CAHIERS DE

NARRATOLOGIE

\section{Cahiers de Narratologie}

Analyse et théorie narratives

27 | 2014

Les bifurcations du récit interactif: continuité ou rupture?

\title{
De l'interactivité du récit au récit interactif
}

Marc Marti and Raphaël Baroni

\section{(2) OpenEdition}

Journals

Electronic version

URL: http://journals.openedition.org/narratologie/7077

DOI: 10.4000/narratologie. 7077

ISSN: 1765-307X

Publisher

LIRCES

\section{Electronic reference}

Marc Marti and Raphaël Baroni, «De l'interactivité du récit au récit interactif », Cahiers de Narratologie [Online], 27 | 2014, Online since 19 December 2014, connection on 22 September 2020. URL : http:// journals.openedition.org/narratologie/7077 ; DOI : https://doi.org/10.4000/narratologie.7077

This text was automatically generated on 22 September 2020 .

Article L.111-1 du Code de la propriété intellectuelle. 


\title{
De l'interactivité du récit au récit interactif
}

\author{
Marc Marti and Raphaël Baroni
}

\section{Les avatars des récits qui bifurquent}

1 À ses origines, la théorie du récit, notamment la narratologie d'inspiration formaliste et structuraliste, s'est développée dans le creuset des études littéraires. De ce fait, le point focal de cette discipline a longtemps été constitué par le récit verbal, généralement sous la forme d'un texte écrit et planifié par un auteur, laissant de côté les formes narratives émergentes, participatives ou interactives. Pourtant, cette restriction de principe à une forme de récit dont le texte constitue le cœur ne correspondait pas au projet des premiers narratologues. En 1964, lorsque Claude Bremond proposa de prolonger les travaux engagés par le formaliste russe Vladimir Propp à partir de l'analyse d'un corpus de récits folkloriques, il souligna d'emblée que sa méthode d'analyse et son objet, le récit, transcendaient les genres et les médias.

Ce que Propp étudie dans le conte russe, [...] c'est une couche de signification autonome, dotée d'une structure qui peut être isolée de l'ensemble du message : le récit. Par suite, toute espèce de message narratif, quel que soit le procédé d'expression qu'il emploie, relève de la même approche à ce même niveau. Il faut et il suffit qu'il raconte une histoire. La structure de celle-ci est indépendante des techniques qui la prennent en charge. Elle se laisse transposer de l'une à l'autre sans rien perdre de ses propriétés essentielles: le sujet d'un conte peut servir d'argument pour un ballet, celui d'un roman peut être porté à la scène ou à l'écran, on peut raconter un film à ceux qui ne l'ont pas vu. Ce sont des mots qu'on lit, ce sont des images qu'on voit, ce sont des gestes qu'on déchiffre, mais à travers eux, c'est une histoire qu'on suit; et ce peut être la même histoire. (Bremond $1964: 4$ )

2 Quelques années plus tard, Todorov créa le néologisme «narratologie » dans le but avoué d'émanciper la jeune théorie du récit de son origine littéraire :

La narration est un phénomène que l'on rencontre non seulement en littérature mais aussi dans d'autres domaines qui pour l'instant relèvent, chacun, d'une discipline différente (ainsi contes populaires, mythes, films, rêves, etc.). Notre 
effort ici sera d'aboutir à une théorie de la narration, telle qu'elle puisse s'appliquer à chacun de ces domaines. Plutôt que des études littéraires, cet ouvrage relève d'une science qui n'existe pas encore, disons la narratologie, la science du récit. (Todorov 1969 : 10)

3 En dépit des intentions affichées, les travaux de Todorov, de Bremond et de leurs successeurs sont longtemps restés confinés à l'analyse de textes littéraires et si l'étude d'autres médias s'est progressivement agrégée à ce courant de recherche, il s'agissait le plus souvent d'objets qui partageaient avec leurs modèles littéraires certaines propriétés essentielles : films et bandes dessinées sont généralement traités comme des fictions dont l'intrigue est agencée par une sorte de "méga-narrateur » (Gaudreault 1999) ou de " grand imagier " (Laffay 1947 ; Boillat 2007 : 322-326) assurant la cohésion et la cohérence du monde raconté.

On observe cependant, depuis une vingtaine d'année, une accélération dans le processus d'élargissement des objets d'étude de la narratologie, accompagnée par une mutation épistémologique. Retraçant cette évolution, Jan Christoph Meister observe ainsi un changement d'orientation faisant passer la théorie du récit de l'étude de phénomènes textuels à l'analyse "des fonctions cognitives de récits oraux et non littéraires, ouvrant ainsi un nouveau chapitre dans le projet narratologique " (2009: 340). Meister insiste en particulier sur l'essor des travaux qui explorent la pertinence des concepts narratologiques pour l'étude des genres et des médias qui s'écartent du modèle constitué par le texte littéraire. Dans cette évolution, les travaux de MarieLaure Ryan ont joué un rôle pionnier, depuis son Possible Worlds, Artificial Intelligence, and Narrative Theory (1991), jusqu'aux plus récents Narrative as Virtual Reality (2001), Narrative across Media (2004), et Avatars of Story (2006). Dans ce dernier ouvrage, MarieLaure Ryan se demande :

comment la narratologie classique, dont le principal objet était, jusqu'à présent, constitué par des textes qui présentent une certaine combinaison de modes diégétique, représentationnel, rétrospectif, scénarisé, réceptif, autonome, déterminé et littéral - peut être étendue à des récits numériques, qui sont des simulations plutôt que de représentations, qui sont émergents plutôt que définis par un script, participatifs plutôt que réceptifs et simultanés plutôt que rétrospectifs. Alors que les textes numériques apportent de nouvelles variations dans les manifestations des catégories narratives traditionnelles que sont le personnage, l'événement, le temps et l'espace, c'est dans le domaine de l'architecture textuelle et dans l'implication de l'utilisateur qu'ils ouvrent véritablement de nouveaux territoires pour l'enquête narratologique. (Ryan 2006 : $\mathrm{xxi}$, notre traduction ${ }^{1}$ )

5 Bien que certains auteurs soulignent le caractère relativement linéaire d'un grand nombre de jeux vidéo, cette problématique de l'architecture textuelle et de l'implication de l'utilisateur deviennent cruciales dans le contexte ludologique (centrée sur l'expérience de l'utilisateur) et dans le cadre des simulations fortement interactives, tels que celles évoquées par Nicolas Szilas dans ce dossier. Ainsi que le souligne ce dernier, ce ne sont pas seulement les théoriciens du récit mais également les créateurs d'univers numériques et les informaticiens qui sont à la recherche de modèles théoriques permettant d'organiser le déroulement des événements simulés sans les mouler dans une intrigue préformatée. On peut toutefois se demander si l'interactivité est une question entièrement nouvelle pour la théorie du récit et si elle dépend fondamentalement de la révolution numérique. 
D'abord, ainsi que le relève Olivier Caïra dans ce dossier (voir aussi Caïra 2011), les fictions interactives ont une longue histoire derrière elles, pour autant que l'on accepte de considérer les pratiques ludiques, notamment les jeux de rôle, comme relevant d'une forme de narrativité fictionnelle émergente. Ensuite, le changement épistémologique évoqué par Meister, qui nous a fait passer de l'étude des textes à l'analyse des fonctions cognitives, a probablement été aussi essentiel que l'élargissement du corpus aux médias numériques pour l'intégration de la problématique de l'interactivité dans le champ de la théorie du récit. En effet, bien avant de s'intéresser aux médias numériques ou aux fictions interactives, certains narratologues avaient déjà intégré dans leurs modèles une forme minimale d'interactivité en évoquant les virtualités de l'intrigue, que l'interprète scénarise au cours sa progression dans le texte. C'est d'ailleurs l'une des principales innovations apportée par Bremond au modèle de Propp, puisqu'il considère que la séquence narrative repose sur un enchaînement de virtualités: 1. possibilité; 2. passage à l'acte ou non-passage à l'acte de cette possibilité ; 3. réussite ou échec de l'action entreprise. Bremond souligne par ailleurs le lien évident entre ces virtualités de l'intrigue et l'intérêt du récit :

Même si le héros triomphe toujours, même si l'auditeur le sait d'avance et l'exige, cette victoire n'a d'intérêt dramatique qu'autant que les chances d'un échec, entrant en concurrence avec la forte finalisation du récit, réussissent à le tenir en haleine jusqu'à la fin du combat. (Bremond $1973: 21$ )

Quelques années plus tard, en appliquant une théorie des mondes possibles à l'étude de la réception des fictions littéraires, Umberto Eco a souligné que la fabula ne se réduisait pas à une propriété immanente du texte ; elle est au contraire une forme en perpétuelle évolution, dont la configuration mouvante dépend de la progression du lecteur :

La fabula n'est pas produite après que le texte a été entièrement lu : la fabula est le résultat d'une série continue d'abductions élaborées au cours de la lecture. Ainsi, nous faisons toujours l'expérience de la fabula étape par étape. (Eco 1979 : 31, n.t.)

Plus proches de nous, les travaux d'inspiration cognitiviste ont poursuivi cette exploration des virtualités du récit en la liant à l'immersion dans le monde fictionnel :

Généralement, l'immersion temporelle consiste en l'implication du lecteur dans le processus par lequel la progression du temps narratif distille le champ du possible, sélectionnant une branche comme réelle et confinant les autres dans le royaume de l'éternelle virtualité ou du contrefactuel, le résultat de cette sélection engendrant continuellement de nouvelles gammes de virtualités. (Ryan $2001: 141$, n.t.)

Dans la même veine, pour Hilary Dannenberg, «la fascination que le lecteur éprouve pour les mondes fictionnels [...] s'explique par le fait que le récit ne se contente pas de raconter une histoire, mais tisse au contraire une toile riche et ontologiquement multidimensionnelle de mondes possibles alternatifs» (Dannenberg $2004: 160$, n.t.). Autrement dit, la dynamique du récit repose sur le désir cognitif du lecteur d'entrer en possession de la configuration finale de l'intrigue, mais cette « constellation cohérente et définitive des événements " ne se révèle (si elle se révèle, ce qui n'est pas certain) qu'à la clôture du récit. En revanche, l'intrigue dans son aspect encore irrésolu est une «matrice de possibilités ontologiquement instable» (Dannenberg $2009: 13$, n.t.).

Reste que cette exploration des virtualités du récit demeure limitée, dans le texte littéraire, par le caractère linéaire et irréversible de la progression du lecteur. Aucun choix ne s'offre véritablement à l'interprète, puisque la branche actualisée par le texte rejette les autres "dans le royaume de l'éternelle virtualité ». Certains considèrent ainsi que c'est la nouvelle de Borges Le Jardin aux sentiers qui bifurquent qui a fourni le 
modèle des fictions véritablement interactives, car dans ce récit, l'auteur évoque un livre imaginaire dans lequel les virtualités ne sont pas seulement esquissées mais font partie intégrante de l'œuvre :

Dans toutes les fictions, chaque fois que diverses possibilités se présentent, l'homme en adopte une et élimine les autres; dans la fiction du presque inextricable Ts'ui Pên, il les adopte toutes simultanément. Il crée ainsi divers avenirs, divers temps qui prolifèrent aussi et bifurquent. De là, les contradictions du roman. Fang, disons, détient un secret; un inconnu frappe à sa porte ; Fang décide de le tuer. Naturellement, il y a plusieurs dénouements possibles : Fang peut tuer l'intrus, l'intrus peut tuer Fang, tous deux peuvent être saufs, tous deux peuvent mourir, et cætera. Dans l'ouvrage de Ts'ui Pên, tous les dénouements se produisent; chacun est le point de départ d'autres bifurcations. (Borges 1965: 100-101)

11 On remarquera cependant que, même dans cette œuvre imaginaire (virtuellement infinie et en réalité impossible), l'initiative n'a pas été véritablement transférée de l'auteur au lecteur, puisque Ts'ui Pên demeure le seul architecte de son labyrinthe. Dans ce cas, l'interaction entre le texte et le lecteur est encore conditionnée par l'architecture imaginée par l'auteur. Dans le prolongement de cet exemple fictif, on peut avancer que la principale limitation pour l'émergence d'un véritable récit interactif tient au présupposé formaliste que l'intrigue devrait nécessairement correspondre à une forme de planification antérieure à l'acte de réception. Or, une telle présupposition est loin d'aller de soi, même pour la littérature la plus classique. Ainsi que le relève Marc Escola, les récits fondés sur une causalité rétrograde (contes, nouvelles), qui ont longtemps constitué le corpus privilégié des narratologues structuralistes, s'opposent au " vaste ensemble des fictions qui se trouvent échapper de facto à la conception téléologique qui veut qu'un roman s'écrive à partir de son dénouement » :

Bien des romanciers de l'âge baroque au siècle des Lumières, comme après eux les «feuilletonistes » et plus près de nous les scénaristes des séries télévisées, écrivent en effet dans l'ignorance de la fin d'une fiction qu'ils livrent au public par livraisons successives (tomaisons, livraisons ou épisodes) et qu'ils poursuivent sans canevas bien établi. Des fictions que l'on dira périodiques, parce qu'elles sont d'abord conçues pour voir le jour par "parties séparées", et qu'elles commencent donc à paraître sans que l'auteur, et moins encore l'éditeur, dispose d'un manuscrit achevé ni d'une idée bien nette des « parties » suivantes et du dénouement de l'intrigue. Le mode de publication décide ici du mode de rédaction, et enveloppe une série de conséquences proprement narratologiques, lesquelles demandent de nouveaux gestes d'analyse dont j'ai essayé de donner des exemples dans une série d'articles consacrés aux longs romans-mémoires inachevés du premier XVIII ${ }^{\mathrm{e}}$ siècle. (Escola 2010 : n.p.)

12 Escola souligne qu'un tel recadrage conceptuel nous oblige donc à redéfinir certains concepts narratologiques fondamentaux. En effet, si l'intrigue ne repose pas sur la planification globale d'un auteur, il faut tenir compte de la possibilité que les lecteurs puissent intervenir sur les choix scénaristiques qui se posent lors du processus d'écriture soumis à la périodicité. Par exemple, une mauvaise audience pour une série télévisée entraînera un dénouement anticipé et l'on sait que les lecteurs de Conan Doyle, ulcérés par le décès tragique de Sherlock Holmes en Suisse, sont parvenus à ressusciter leur héros en contraignant l'auteur à poursuivre une série à laquelle il voulait mettre un terme. Parfois, les auteurs de feuilletons sont allés jusqu'à solliciter directement les avis de leurs fans, sondant leurs opinions par la médiation du courrier des lecteurs ou des fanfictions, de sorte que ces derniers ont pu avoir l'impression de 
décider de la suite de l'histoire. Aujourd'hui, un réseau comme Netflix décide du genre de série qu'il produira sur la base de statistiques liées aux utilisateurs de son site.

La révolution numérique a accéléré ce mouvement de convergence entre les usagers et les producteurs de mondes fictionnels dont Henry Jenkins (2013) a décrit la logique économique et les étapes historiques. Ainsi que l'illustre dans ce dossier l'article de Cyril Bornet, de Daniel de Roulet et de Frédéric Kaplan, le passage du livre à différentes formes d'interfaces numériques, offre par exemple de nouvelles possibilités au lecteur pour reconfigurer l'intrigue du roman. Mais il faut admettre que la forme narrative, même quand elle se présente sous la forme d'un texte littéraire publié dans un livre, n'est pas toujours aussi planifiée, linéaire et monogérée que ce que les modèles formalistes ont pu nous laisser supposer. Si l'intrigue peut être décrite - en adoptant un point de vue fonctionnaliste et cognitiviste - comme une matrice de possibilités ontologiquement instable, comme un dispositif dont la fonction première est d'ouvrir des virtualités narratives et d'engendrer de la curiosité ou du suspense (Baroni 2007 ; 2009), alors il n'y a pas de raison de la considérer comme un obstacle à l'analyse de récits émergents et participatifs, voire de simulations ou de pratiques ludiques hautement interactives. Ainsi que l'affirmaient les premiers narratologues, le récit est une forme qui transcende les médias et si l'on peut reconnaître ses " avatars » jusque dans les jeux de rôle et les jeux vidéo, alors la confrontation entre narratologie et ludologie peut s'avérer une étape incontournable dans la refondation des concepts de la théorie du récit. Ainsi que l'affirmait récemment Werner Wolf: "une approche intermédiale peut [...] contribuer à éviter les généralisations unilatérales que l'on a pu observer dans les recherches mono-médiales antérieures" en particulier dans les travaux «centrés sur les textes verbaux" (Wolf 2003 : 193, n.t.). En retour, on peut espérer que ce recadrage conceptuel offre un point de vue nouveau sur les phénomènes narratifs, dont l'impact pourrait aller jusqu'à transformer notre appréhension des romans de l'âge baroque jusqu'aux fictions télévisuelles qui dominent le paysage médiatique contemporain.

\section{Narratologie vs ludologie}

14 La ludologie est aussi ancienne que la narratologie. Dès le départ, la ludologie s'est affirmée comme une science des jeux qui prenait en compte à la fois l'organisation de la pratique ludique (espace, temps, règles), mais aussi sa dimension sociale (position du joueur, effets du jeu). Ses fondements remontent aux travaux d'Huizinga (1938) et son intérêt de philosophe et historien pour l'homo ludens. Dans sa théorie, quelques éléments peuvent retenir l'attention, comme le souligne Colas Duflo, soit :

a. Le libre consentement du joueur : là où il y a obligation (sociale) il n'y a pas jeu.

b. Le jeu est limité temporellement et spatialement.

c. Tout jeu est doté de règles propres qui n'ont du sens qu'à l'intérieur du jeu.

d. Le jeu est « improductif ».

(Duflo 1997 : 37-38)

Quatre décennies plus tard, Roger Caillois reprenant le travail d'Huizinga définit le jeu comme une activité :

$1^{\circ}$ - libre : à laquelle le joueur ne saurait être obligé sans que le jeu perde aussitôt sa nature de divertissement attirant et joyeux.

$2^{\circ}$ - séparée : circonscrite dans des limites d'espace et de temps précises et fixées à

l'avance. 
$3^{\circ}$ - incertaine : dont le déroulement ne saurait être déterminé ni le résultat acquis préalablement sans une certaine latitude dans une nécessité d'inventer étant obligatoirement laissée à l'initiative du joueur.

$4^{\circ}$-improductive : ne créant ni bien, ni richesse, ni élément nouveau d'aucune sorte ; et, sauf déplacement de propriété au sein du cercle des joueurs, aboutissant à une situation identique à celle du début de la partie.

$5^{\circ}$ - réglée : soumise à des conventions qui suspendent les lois ordinaires et qui instaurent momentanément une législation nouvelle qui seule compte.

$6^{\circ}$ - fictive : accompagnée d'une conscience spécifique de réalité seconde ou de franche irréalité par rapport à la vie courante.

(Caillois $1967: 42$ )

Il indique que «ces diverses qualités sont purement formelles. Elles ne préjugent pas $\mathrm{du}$ contenu des jeux. Il propose ensuite une typologie des jeux en fonction de l'effet que ceux-ci produisent sur le joueur en distinguant différentes impulsions primaires (Caillois 1967 : 47-48). Celles-ci renvoient à la compétition (agôn) au destin (alea), au mimétisme ou faire-semblant (mimicry) et au vertige (ilinx). Ces impulsions se distribuent, en fonction des jeux et des moments de jeu, entre deux pôles :

a. le ludus, pour les jeux qui sont régis par un nombre de règles très important ;

b. le paidia, pour ceux qui laissent une grande marge de manœuvre au joueur et donc à l'improvisation.

(Caillois $1967: 48$ )

17 Avant l'arrivée du jeu vidéo, les contacts entre narratologie et ludologie étaient pour le moins limités. En premier lieu, les passerelles entre le monde du jeu et celui des textes narratifs n'apparaissaient pas comme une évidence. Ensuite, la narratologie, comme nous l'avons rappelé, s'est longtemps cantonnée à l'analyse des phénomènes textuels préconfigurés en amont par les producteurs des récits, alors que la ludologie s'intéressait bien plus aux usages et effets du jeu qu'aux schémas narratifs qu'ils contenaient. Cependant, parallèlement aux évolutions de la narratologie, la révolution numérique a considérablement modifié la donne. L'arrivée dans le champ culturel de nouveaux objets, dont les recherches en sciences humaines se sont emparées, est venue remettre en question les limites méthodologiques inhérentes aux deux approches. La ludologie et la narratologie se sont avérées insuffisantes pour rendre compte du jeu vidéo dans son ensemble. Comme le rappelle Julien Rueff (2008:147), c'est le concept de games studies, englobant de très nombreux types d'approches, qui s'est imposé pour dépasser ces limites. Les games studies se définissent comme un ensemble interdisciplinaire dans lequel s'interpénètrent les méthodes de disciplines constituées (histoire, sociologie, psychologie, anthropologie) et de disciplines transversales (génétique textuelle, sémiotique, narratologie et ludologie). Rueff examine ensuite les divergences de départ entre ludologie et narratologie, qui pourraient se résumer par la formule homo ludens vs homo fabulator. Les narratologues supposent que :

les êtres humains utiliseraient la narration dans toutes [leurs] pratiques et [leurs] représentations, et les jeux vidéo ne feraient pas exception. Ensuite, ces loisirs numériques comporteraient de nombreux éléments narratifs évidents (leur scénario, leur introduction cinématique, etc.). Enfin, il existerait de nombreuses similarités entre les jeux vidéo et les narrations. (Rueff 2008 : 148-149)

Quant aux ludologues, ils insistent sur les restrictions de l'approche narratologique en reconnaissant :

l'existence possible d'éléments narratifs dans les jeux vidéo. Cependant, ils contestent la légitimité de la narratologie, en soulignant les insuffisances d'une 
approche exclusivement centrée sur les aspects narratifs, aveugle aux spécificités

de ces loisirs numériques. (Rueff 2008 : 148-149)

19 Ces deux positions résument assez bien le problème tel qu'il a été posé au départ. Cependant, l'opposition peut être largement dépassée si l'on pratique, dans un esprit interdisciplinaire tel que le préconisait Edgar Morin, la migration des concepts entre les disciplines et les méthodes (Morin 1990). Il convient de ne pas oublier que chaque discipline travaille sur des objets qu'elle a préalablement construits. L'apparition du jeu vidéo est sans doute venue remettre en question la limite de l'objet «jeu » (construit par la ludologie) tout comme il a remis en question le "récit» (construit par la narratologie). Dans le cadre des travaux présentés dans ce numéro de revue, les contributeurs se sont essayés à redessiner les contours de leurs objets, ainsi que l'approche de la dimension narrative du jeu à partir de leur discipline (narratologie, histoire, modélisation informatique, sociologie). Cette introduction n'a pas pour ambition de cerner tous les apports de la ludologie à la narratologie, mais de fournir quelques illustrations de la manière dont l'apparente opposition peut être dépassée.

La typologie de Roger Caillois peut éclairer l'analyse narratologique, mais la narratologie peut aussi contribuer à perfectionner l'analyse ludologique. Si l'on considère les impulsions primaires, on peut observer par exemple que la mimicry dans le jeu vidéo dépend de nombreux facteurs narratifs. Comme l'analysent Marc Marti et Selim Krichane dans ce dossier, la péritextualité narrative (jaquette, affiches, bandes annonces narratives, cinématiques internes) contribue à l'immersion mimétique du joueur et renforce sans nul doute la mimicry. Dans ce cadre aussi, le choix de l'avatar ou bien le moment pendant lequel le joueur « endosse » son identité ludique peuvent être analysés à partir des deux points de vue. En effet, un avatar possède des fonctions narratives différentielles qui permettront de réaliser ou pas certaines actions dans le récit vidéoludique. Par ailleurs, l'avatar renforce aussi considérablement la mimicry, même si la différenciation entre l'avatar et le sujet joueur est maintenue (Genvo 2009).

Dans le cas des FPS (First Person Shooter, jeu de tir à la première personne), on peut remarquer que le récit mis en place par le jeu dépend du choix de l'utilisateur qui peut le joueur de plusieurs façons. En effet, dans un premier temps (celui de la découverte), la plupart des joueurs essaient de terminer le jeu, clôturant ainsi le récit et privilégiant donc mimicry et ludus. Cependant, dans un second temps, ces jeux ont souvent une " seconde vie ", car ils servent de supports à des tournois en ligne ou des parties contre l'intelligence artificielle, qui relèvent plutôt de l'agôn et du paidia et qui permettent de produire des récits d'un autre genre. Selon la ludogie, en fait, ce type de jeu vidéo serait à la fois des jeux, quand le joueur est plongé dans une partie, mais en même temps des espaces ludiques qui offrent une palette de jeu assez variée, de la même façon qu'un stade ou un gymnase sont adaptés à un grand nombre de sports différents. Un certain nombre de phénomènes narratifs gagneraient à être identifiés avec des notions de la ludologie, qui possèdent l'avantage de renvoyer au joueur et à ses « impulsions » selon la terminologie de Caillois, une catégorie pour laquelle la narratologie reste sans doute plus démunie.

\section{Présentation du dossier}

22 Ce numéro des Cahiers de narratologie est issu d'une journée d'étude qui s'est tenue à l'Université de Lausanne (UNIL) le 14 février 2014. Cette journée était organisée par 
Raphaël Baroni, Marc Marti, Claire Clivaz et Frédéric Kaplan et elle a bénéficié du soutien de l'EFLE (UNIL), du LADHUL (UNIL), du LIRCES (Université de Nice Sophia Antipolis), du DHLAB (Ecole Polytechnique Fédérale de Lausanne) et du Réseau romand de narratologie (RRN : www.narratologie.ch).

L'article de Cyril Bornet, Daniel de Roulet et Frédéric Kaplan qui ouvre le numéro présente une expérience de création et de lecture narrative interactive sur le Net à partir de La Simulation humaine, roman fleuve écrit par Daniel de Roulet. La forme choisie et proposée en ligne http://simulationhumaine.tk/ offre les perspectives d'une expérience originale de création et de réception nouvelle pour une œuvre narrative. L'originalité de cette recherche participative repose sur une «recombinaison narrative " à partir d'outils d'écriture et de lecture numérique. Le lecteur se voit proposer de lire soit l'intégralité du roman (de façon classique) soit des " morceaux » de durées inégales, mais qui présentent chacun la particularité de pouvoir être lus de façon autonome grâce à une combinatoire qui en assure la logique. On retrouve ici, à l'origine, la métaphore de la Rayuela de Julio Cortázar, mais nettement amplifiée par la technologie et la réflexion des concepteurs de La simulation humaine, dans une expérience originale qui ouvre des portes à de nouvelles théories de la réception. Le rapprochement du roman fleuve interactif avec les séries télévisées permet par exemple aux auteurs de retenir un certain nombre de facteurs nécessaires à son fonctionnement, comme la narration en forme de flot, un effet d'accroche continuel et la promesse de dénouements à long terme qui permettent d'entretenir le désir de lire les «épisodes » suivants. Par ailleurs, la possibilité de suivi statistique qu'offre le support (que lisent réellement les lecteurs et comment?), bien qu'encore relativement nouvelle, offre la perspective à moyen terme de pouvoir " améliorer » la recombinaison narrative pour la rendre plus attractive. A plus long terme, les auteurs espèrent pouvoir entamer une réflexion sur les nouveaux outils qui pourraient être proposés aux écrivains afin de leur permettre de tirer parti de nouveaux artefacts d'écriture.

Le travail d'olivier Caïra se positionne comme une socio-narratologie des jeux. Il développe principalement des comparaisons entre les jeux « analogiques » et les jeux numériques. Les jeux analogiques intègrent des dimensions d'immersion et d'interactivité que l'on a tendance à considérer comme l'apanage des supports numériques. La narratologie peut y trouver un objet d'étude original, en particulier parce ces jeux font appel à d'autres ensembles sémiotiques que le langage verbal écrit, principalement la gestuelle et le langage oral, qui articulent de véritables storygames. L'article présente aussi l'intérêt de revenir sur un certain nombre de stéréotypes narratologiques auxquels est sujette la notion d'interactivité, souvent cantonnée au monde numérique, alors que celui-ci tend à la capturer dans des contraintes narratives plus ou moins fortes, en particulier à travers la scénarisation programmée. En revanche, le jeu "analogique» se caractérise par une interaction plus grande (en langue naturelle, par la gestuelle, par le dessin) qui offre une scénarisation toujours ouverte, dans laquelle les possibles narratifs dépendent de l'interaction entre les participants et surtout restent en partie imprévisibles. Cependant, l'analogique comme le numérique semblent répondre à une "écologie de l'intrigue ", c'est-à-dire un déroulement dans un espace-temps (une diégèse) qui va sur-déterminer l'action suivant l'intervention des joueurs. La diégèse telle que la définit Olivier Caïra devient un foyer de tension narrative, ce qu'il nomme " les tenseurs environnementaux » indépendants 
de l'action à proprement parler, mais toujours potentiellement actifs comme matrices d'intrigues.

Le texte de Selim Krichane envisage l'évolution de l'intrigue vidéoludique dans les années 1990 et engage un travail historique des plus intéressants, d'autant que cette approche reste encore relativement rare pour un objet qui s'approche du demi-siècle d'existence. La prise en compte des évolutions technologique peut s'avérer d'une grande utilité lorsque l'on s'intéresse à la narrativité. Par exemple, des éléments autrefois statiques (et souvent textuels) ont progressivement été intégrés à la narration numérique et au jeu, comme les cinématiques ou la disposition d'une information à l'intérieur des niveaux. L'analyse propose ainsi d'observer au cours de cette décennie comment les jeux ont évolué entre la présentation figée d'un récit-cadre d'une part, et l'actualisation d'un récit émergent d'autre part. Parmi les phénomènes caractéristiques, l'article étudie les films interactifs, l'évolution des jeux de combat spatial et les jeux de rôle. La partie consacrée à la vision (que la narratologie qualifierait de focalisation) insiste sur le rôle majeur joué par l'utilisation de la 3D polygonale et "l'invention» d'un nouveau mode de narration, dans lequel la découverte de l'espace prend une importance primordiale. La conclusion insiste sur le rôle clé joué par les années 1990, où l'on voit « apparaître une tendance à morceler et à disperser des informations narratives au sein des séquences interactives, notamment par l'utilisation de la voix des personnages ». Cette tendance démontre la dissolution progressive, pour ne pas dire la disparition, des informations narratives figées. Celles-ci viennent s'intégrer à la dynamique de la progression du jeu, en étant non plus révélées en bloc, mais au fur et à mesure de l'avancée de la narration, contribuant ainsi au maintien de la tension narrative.

Marc Marti revient dans un premier temps sur l'opérativité de la notion de genre appliquée au jeu vidéo à partir des travaux de Dominique Arsenault (Arsenault 2011). La notion de genre peut permettre d'envisager l'univers vidéoludique en se fondant sur des prototypes narratifs. Ainsi, le jeu vidéo se caractériserait par des degrés de narrativité plus ou moins importants en fonction du genre auquel il appartient ou en fonction de certains moments du développement de la partie. Cette distinction des prototypes permet de postuler une immersion du joueur de nature différente en fonction du degré de narrativité, en distinguant l'endo-narratif du narratif. De façon schématique, une faible narrativité correspondrait à une immersion physique amplifiée (de l'ordre de l'endo-narratif) alors que la forte narrativité privilégierait l'immersion psychologique (de l'ordre du narratif). Finalement, l'immersion est définie comme reposant sur "un effet de monde ", qui procède d'une diégèse, incluant le référent spatio-temporel de l'intrigue et des éléments de suspense ou de curiosité qui sont mis en œuvre. Dans la dernière partie, Marc Marti ébauche une exploration du nouveau statut narratif du joueur. Au-delà de l'expérimentation des récits contrefactuels que permettent tous les jeux dans leur pratique, il apparaît que, ces dernières années, un nombre de joueurs non négligeable se retrouve dans la position d'auteurs de récits de jeu. Ce phénomène méta-narratif, encore peu exploré, contribue pourtant à modifier considérablement la façon dont les jeux vidéo sont perçus et pratiqués.

L'article de Marie-Laure Ryan s'intéresse aux jeux et récits numériques à partir de l'intensification de l'expérience spatiale qu'ils proposent. Le jeu vidéo se construit ainsi à partir de deux typologies spatiales: l'espace émotionnel ou affectif et l'espace stratégique, qui suppose la mise en œuvre d'actions. Dans sa dimension émotionnelle, 
l'espace vidéoludique permet au joueur de projeter des émotions et d'acquérir des expériences. La forme limite de cette modalité étant incarnée par des mondes virtuels tels que Second life. L'espace stratégique se définit au contraire comme un terrain de jeu organisé de manière abstraite, ce qui apparait de façon évidente dans les premiers jeux tels que Pac-man. Dans un tel espace, le joueur sait qu'il doit avant tout apprendre les règles spécifiques au jeu dans lequel il est engagé. Cependant, l'évolution technologique (graphismes et interfaces) fait que cette dimension « abstraite » a reculé, car l'espace et la façon de s'y mouvoir sont devenus plus concrets. L'expérience pratique du monde réel devient alors en partie transposable dans l'activité vidéoludique. Ainsi, l'espace simulé combine des dimensions stratégiques et mimétiques. Par ailleurs, l'espace se présente sous deux modalités, la route et le monde. Ces deux métaphores permettent de caractériser la linéarité narrative des jeux de type aventure ou FPS, qui fonctionnent par accumulation et progression, à laquelle s'oppose la "promenade » qui épouse la logique des jeux-monde. Finalement, si on prend en compte à la fois les dimensions stratégiques et émotionnelles, ainsi que les trajets (routes) ou les promenades (mondes), «les mondes des jeux numériques ne sont pas seulement une arène où les joueurs démontrent leur habileté, ils peuvent aussi devenir un habitat qui inspire le sentiment d'appartenir à une communauté ». En conclusion, Marie-Laure Ryan souligne que ce qui fait la spécificité des médias numériques, c'est l'expérience de l'espace et non l'expérience temporelle, pour laquelle ils sont globalement similaires aux médias traditionnels.

Nicolas Szilas propose un point de vue intéressant ancré à la fois dans son expérience de concepteur de récit interactif et de théoricien du jeu. Par une approche empirique fondée sur trois exemples, il propose d'ébaucher une typologie des intrigues dans le récit fortement interactif, tout en considérant la qualité des intrigues selon des critères distincts et spécifiques. Comme point de départ, l'article pose la question de la définition de ce que serait le récit fortement interactif. La possibilité de faire reposer cette notion uniquement sur le développement de toutes les possibilités narratives ne semble pas assez pertinente. L'auteur propose donc de définir ce type de récits à partir de trois objectifs spécifiques, soit :

1. mettre en scène « des événements qui sont le résultat d'un calcul, tant au niveau de leur occurrence (si ça arrive et quand ça arrive), leur contenu (acte narratif) que leur formulation";

2. donner « à l'utilisateur le rôle d'un personnage principal de l'histoire ; »

3. induire «chez l'utilisateur une expérience qu'il qualifie de narrative ».

Du point de vue de la conception, les intrigues écrites (principales ou secondaires) sont distinguées des intrigues résultantes. Les premières sont pensées par l'auteur et écrites par lui-même. Bien que différant techniquement des intrigues romanesques par leur format, elles supposent cependant une linéarité des événements. Elles possèdent par ailleurs trois spécificités : elles sont potentiellement multilinéaires (développement des possibles narratifs), elles acceptent potentiellement dans leur déroulement des intrigues secondaires et elles sont susceptibles de changements de prise de vue. Dans une interactivité forte, le programme doit pouvoir insérer des intrigues secondaires à plusieurs moments du jeu. Celles-ci restent secondaires car leur déclenchement n'est pas obligatoire pour parvenir au terme de la partie. Les intrigues résultantes se définissent par leur génération, qui n'est pas directement liée au choix de l'auteur mais qui résulte du calcul effectué par le programme, qui va agencer des événements dans 
un certain ordre en fonction des choix du joueur. Nicolas Szilas distingue ici les intrigues contrôlées et les intrigues résultantes. Les premières sont constituées par «l'arrangement des événements de l'intrigue [qui] a été calculé par le système, selon des règles conçues pour constituer une intrigue, au sens large, c'est-à-dire selon des principes d'ordre narratif ». Dans les secondes, c'est le hasard qui vient établir « une suite d'événements, dont la succession n'est ni narrativement calculée par la machine ni explicitement écrite par l'auteur, [et qui] constitue une intrigue ». Pour préciser la terminologie actuellement en vogue, le terme d'intrigue émergente est relativement imprécis et, dans ce cas, l'auteur propose plutôt d'utiliser le terme d'intrigue "détournée", que l'on retrouve de façon récurrente dans les pratiques ludiques actuelles. Les spécificités analysées amènent Nicolas Szilas à repenser en termes plus spécifiques la qualité de l'intrigue interactive, qui n'est pas entièrement comparable à celle des médias antérieurs, en particulier parce que l'interaction avec un récit diffère de la perception de ce récit quand il est impossible d'agir sur son déroulement, même si les événements qui se succèdent sont les mêmes. Dans l'interactivité, il semble bien que " l'intrigue réussie sera celle qui saura au mieux gérer les sentiments de compétence et de frustration ", qu'il convient de rapprocher des études sur l'apprentissage et les jeux pédagogiques. De plus, ce type de jeu diffèrerait des autres genres vidéoludiques car « la compétence concernée n'est ni sensori-motrice comme dans les jeux d'action, ni logico-mathématique ». Celle-ci «serait plutôt socio-psychologique, car le récit, par définition, met en œuvre des personnages dans leur quête et leur transformation ».

Le prochain numéro des Cahiers de narratologie, qui sera consacré au récit comme acte cognitif, prolongera dans un certain sens les travaux publiés dans ce volume.

\section{BIBLIOGRAPHY}

\section{Références}

Arsenault, Dominic (2011), Des typologies mécaniques à l'expérience esthétique Fonctions et mutations du genre dans le jeu vidéo, Montréal, Thèse en ligne, URL : https://papyrus.bib.umontreal.ca/ xmlui/bitstream/handle/1866/5873/Arsenault_Dominic_2011_these.pdf?sequence=2

Baroni, Raphaël (2007), La tension narrative, Paris, Seuil, coll. « Poétique ».

Baroni, Raphaël (2009), L'œuvre du temps, Paris, Seuil, coll. « Poétique ».

Boillat, Alain (2007), Du bonimenteur à la voix-over. Voix-attraction et voix-narration au cinéma, Lausanne, Antipodes.

Borges, Jorge Luis (1965), Fictions, Paris, Gallimard, coll. « folio ».

Bremond, Claude (1964), «Le message narratif », Communications, vol. 4, n 4, p. 4-32.

Bremond, Claude (1973), Logique du récit, Paris, Seuil, coll. « Poétique ».

Caillois, Roger (1967), Les jeux et les hommes, Paris, Gallimard, [1958]

Caïra, Olivier (2011), Définir la fiction, Paris, Editions de l'EHESS. 
Dannenberg, Hilary (2004), « Ontological Plotting : Narrative as a Multiplicity of Temporal Dimensions ", in The Dynamics of Narrative Form. Studies in Anglo-American Narratology, J. Pier (dir.), Berlin \& New York, Walter de Gruyter, p. 159-189.

Dannenberg, Hilary (2008), Coincidence and Counterfactuality. Plotting Time and Space in Narrative Fiction, Lincoln, University of Nebraska Press.

Duflo, Colas (1997), Jouer et philosopher, Paris, PUF.

Eco, Umberto (1984), The Role of the Reader, Bloomington, Indiana University Press.

Escola, Marc (2010), «Le clou de Tchekhov. Retour sur le principe de la causalité régressive », Fabula, Atelier de théorie littéraire, consulté le 28 novembre 2014. URL : http://www.fabula.org/ atelier.php?Principe_de_causalite_regressive Gaudreault, André (1999), Du littéraire au filmique. Système du récit, Paris, Armand Colin. Genvo, Sébastien (2011), «Jeux vidéo », Communications vol. 1, n 88, consulté le 4 décembre 2014. URL : www.cairn.info/revue-communications-2011-1-page-93.htm Genvo, Sébastien (2009), «Le rôle de l'avatar dans la jouabilité d'une structure de jeu vidéo », Adolescence, vol. 3, n69, consulté le 4 décembre 2014. URL : www.cairn.info/revueadolescence-2009-3-page-645.htm Jenkins, Henry (2013), La Culture de la convergence. Des médias au transmédia, Paris, Armand Colin. Huizinga, Johan (1988 [1938]), Homo ludens, essai sur la fonction sociale du jeu, Paris, Gallimard. Laffay, Albert (1947), « Le récit, le monde et le cinéma », Les Temps modernes, n²1, p. 1578-1600. Meister, Jan Christoph (2009), « Narratology », in Handbook of Narratology, Peter Hühn, John Pier, Wolf Schmid and Jörg Schönert (dir.), Berlin et New York, De Gruyter, p. 329-350.

Rueff, Julien (2008), « Où en sont les “game studies” ? ", Réseaux, vol. 5, n 151, p. 139-166, consulté le 4 décembre 2014. URL : www.cairn.info/revue-reseaux-2008-5-page-139.htm Ryan, Marie-Laure (1991), Possible Worlds, Artificial Intelligence, and Narrative Theory. Bloomington : Indiana University Press.

Ryan, Marie-Laure (2001), Narrative as Virtual Reality. Baltimore and London : Johns Hopkins University Press.

Ryan, Marie-Laure (dir.) (2004), Narrative across Media, Lincoln, University of Nebraska Press. Ryan, Marie-Laure (2006), Avatars of Story, Minnesota, University of Minnesota Press.

Todorov, Tzvetan (1969), Grammaire du Décaméron, The Hague, Paris, Mouton.

Wolf, Werner (2003), « Narrative and Narrativity : A Narratological Reconceptualization and its Applicability to the Visual Arts », Word and Image, n 19 (3), p. 180-197.

\section{NOTES}

1. Dorénavant, n.t. 